\title{
La academia ante el periodismo post-industrial. De la cultura masiva a la desmasificación y fragmentación
}

Recibido: 27 de noviembre de 2012

Aceptado: 26 de junio de 2013

Publicado: 29 de noviembre de 2013

\author{
Daniel Mazzone \\ mazzone@ort.edu.uy
}

Universidad ORT (Uruguay)

\begin{abstract}
Resumen: El campo comunicacional arrastra una crisis de incorrespondencia entre la acumulación teórica y la reproducción del conocimiento en las carreras académicas de subcampos como el periodismo. Hace tres décadas, Verón y Martín-Barbero instaron a desplazar el análisis de los medios hacia las mediaciones, Scolari (2008) puso en foco las hipermediaciones, esa "trama de reenvíos, hibridaciones y contaminaciones" y Castells (2009), las transformaciones sociales provocadas por la "autocomunicación de masas". Mientras el desafío es pensar en términos de desmasificación y fragmentación, nociones superadas como cultura masiva o industria cultural, resisten y reducen la profesión al monólogo que incide cada vez menos en la gestión informativa. Este trabajo se interroga sobre las condiciones que distorsionan la articulación entre dos planos académicos necesariamente interdependientes.
\end{abstract}

Palabras clave: Ciberperiodismo, academia, medios, mediaciones, hipermediaciones, ecosistema, conocimiento, comunicación, teoría, acumulación, fragmentación.

Abstract: In the academic careers of subfields like Journalism, the communicational field is at a crisis caused by the gap between theory accumulation and knowledge reproduction. Thirty years ago, Verón and Martin-Barbero proposed a new approach, displacing analysis of the media to mediations. In 2008, Scolari started focusing in the hypermediations, consisting of forwards, hybridization and contaminations. In 2009, Castells put emphasis on the observation of social transformations provoked by mass auto-communication. While we must think in dismassification and fragmentation, obsolete notions like massive culture or cultural industry resist, reducing the profession to a poor monologue with little 
incidence in the informative management. The subject of this paper is to discuss the factors that disturb the connection between two academic plans necessarily interdependent.

Key words: Cyberjournalism, Academy, Media, Mediations, Hypermediations, Ecosystem, Knowledge, Communication, Theory, Accumulation, Fragmentation.

"En la edad del conocimiento, no reconocer al otro en su inteligencia, es negar su verdadera identidad social, es alimentar su resentimiento y su hostilidad, es sustentar la humillación, la frustración de la que nace la violencia"

(Pierre Lévy).

\section{Introducción. Dos décadas de ciberperiodismo en medio de la lógica analógica}

Dos intensas décadas de ciberperiodismo no lograron desplazar la lógica analógica que (de mantenerse la tendencia inercial) seguirá hegemonizando las carreras de periodismo en Iberoamérica. Los analistas del campo disciplinar de la comunicación reiteran que la selección de objetos de estudio y de investigación, así como los planes y programas de estudio en países de la región, no condicen con el desarrollo alcanzado por la teoría.

Vasallo y Fuentes (2000), Pereira (2005), Mata (2005), Said (2010) y Mellado (2007 y 2010) coinciden en que la crisis del campo es profunda y no debe abordarse con criterios reduccionistas. Las prácticas y los programas académicos presentan un desfase con las operaciones que las nuevas tecnologías posibilitan, en una distorsión que expande la brecha entre aquello que los usuarios demandan y lo que la academia debería explicar y no explica.

Los análisis coinciden en que el desequilibrio entre teoría y práctica se origina en los mecanismos académicos que deberían operar y no operan para que el ámbito de la reproducción del conocimiento se nutra convenientemente del conocimiento científico. Reiterados desde hace más de una década, los diagnósticos revelan un desfase entre la acumulación teórica y las prácticas académicas, que parecería explicar el lento e insuficiente recambio en las carreras de periodismo, que no terminan de ubicarse a la altura de las innovaciones introducidas por la cultura de las redes reticulares.

Esa distorsión determina el enfoque (a menudo errático) de los cursos, así como la inclusión del periodismo digital a través de materias aisladas e inconexas, cuyo destino se diluye en medio de carreras creadas, en su mayoría, en tiempos pre-digitales y que intentan adaptarse a través de modificaciones parciales en ciclos variables, sin revisiones profundas y a través de materias no troncales desconectadas entre sí. Incluso las escasas materias referidas al ciberperiodismo tienden a centrarse en las nuevas competencias técnicas, con prescindencia de los contenidos teóricos, o incluyendo la teoría de tal modo que difícilmente podría conducir a la comprensión de los procesos mediáticos en curso. 
Esta desvinculación entre la acumulación teórica y las prácticas académicas se ubica en el marco más amplio del proceso de cambios que atraviesa el modelo de educación superior en el siglo XXI, que Gibbons (1998: i) describe como "el surgimiento de un sistema distribuido de producción de conocimiento":

"En lo que a las universidades se refiere, la modificación más profunda es que la producción y divulgación del conocimiento -la investigación y la enseñanza- ya no son actividades autónomas, que se llevan a cabo en instituciones relativamente aisladas. Ahora implican una interacción con otros diversos productores de conocimiento. En estas circunstancias, las vinculaciones entrañarán más y más el aprovechamiento del potencial de las nuevas tecnologías de información y comunicación".

El recorte del alcance cognitivo que proyecta la desarticulación de dos planos académicos que requieren imbricación profunda, distorsiona la adecuada elaboración académica sobre la naturaleza del cambio y la correspondiente divulgación a través de los nuevos profesionales. En consecuencia, las audiencias, el conjunto social, continúa manejándose (como veremos) con concepciones y nociones teóricas superadas, mientras el uso generalizado de los nuevos dispositivos, generadores globales de cambios sociotécnicos, perfila la nueva realidad que modifica las formas de vivir de esas mismas audiencias. Algunos debates públicos, incluso académicos, revelan la brecha evidente entre el consumo mediático en expansión y la comprensión de sus capacidades operativas.

\subsection{Una recepción contradictoria}

Parece por lo menos contradictorio que mientras significativos segmentos de usuarios se incorporan con naturalidad y autonomía crecientes a la generación de circuitos y comunidades virtuales (así como a un nuevo y espontáneo relacionamiento con los medios) al mismo tiempo surjan voces (incluso académicas) que resuciten antiguos temores amenazadores sobre la pretendida capacidad manipuladora de los medios, que podrían manipular unos medios que, si son tradicionales, están constreñidos a resolver su supervivencia a cortísimo plazo, y si son nuevos, se rigen por las reglas culturales de la desmasificación y la fragmentación.

Estas falsas polémicas ya eran señaladas por García Canclini (1991: 6) desde el prólogo al clásico de Martín-Barbero, De los medios a las mediaciones:

"Si los primeros investigadores de medios trataban de saber cómo hacen estos para manipular las audiencias, al estudiar la reformulación del aura artística en la gran ciudad y el proceso de formación de lo popular en las novelas de folletín, la prensa y la televisión [este libro] ofrece una de las refutaciones teóricas más consistentes a las ilusiones románticas, al reduccionismo de tantos marxistas y al aristocratismo frankfurtiano".

La principal dificultad de enfoque de los planes y programas de estudio de periodismo consiste en que las concepciones predominantes se encuentran mayoritariamente fijadas en 
el eje de los medios, con frecuencia todavía percibidos como meros soportes para transportar contenidos. La incomprensión subyacente de la etapa actual de los cambios mediáticos, dificulta la puesta en foco de las operaciones complejas que se realizan desde los nuevos dispositivos y los cambios sucesivos que continúan procesándose.

La ya antigua noción de las mediaciones resulta insuficiente para abordar el nuevo estadio de las hipermediaciones, cuya trama de reenvíos, hibridaciones y contaminaciones (Scolari, 2008) conduce a analizar el nuevo ecosistema de los medios en términos de desmasificación y fragmentación.

\subsection{Un marco analítico y metodológico}

Este trabajo, basado en un relevamiento de la bibliografía especializada, en los resultados de dos investigaciones relativamente recientes (Said en Colombia y Mellado en Chile) y en una observación propia y actual sobre los planes de estudio de Periodismo de las cuatro universidades uruguayas que ofrecen carreras del ramo, se propone relevar y describir el estado de situación del ciberperiodismo en las prácticas académicas en general y en los programas de estudio en particular.

Se pretende ubicar la crisis del campo comunicacional en una doble transición: aquella en la que se encuentra la universidad debido al cambio en las modalidades de producción del conocimiento en el siglo XXI (Gibbons) y, a su vez, en la transición específica que atraviesan la comunicación y el periodismo académicos, en sus intentos (por el momento insuficientes) por incorporar a sus planes y programas de estudio el nuevo escenario de las redes reticulares y los cambios culturales que provoca.

Luego de esta introducción, en el segundo punto, se abordará el marco de crisis en el que se encuentra el campo comunicacional, a partir del giro epistemológico no metabolizado de la década de 1980, en que Eliseo Verón y Jesús Martín-Barbero instaban a superar el enfoque en los medios para pasar a la mediatización y a las mediaciones. Se considerarán algunas consecuencias visibles de esta dificultad en los planes y programas.

En un tercer punto, se procurará examinar la desarticulación teórico-práctica del campo, a partir de los déficits de la divulgación académica y cómo se manifiesta esa falta en la construcción social de la realidad. Algunos diagnósticos indican que al resignar espacios, y operar como espectadora de los cambios, la academia forma profesionales que difícilmente estén en condiciones de introducir prácticas innovadoras, dadas las nociones superadas largamente por la teoría e inoperantes para este momento social en que han adquirido su expertise.

Finalmente, antes de arribar a la sección conclusiva, en el cuarto punto se abordarán algunas de las características del momento comunicacional actual, como las que imponen las hipermediaciones, caracterizadas por la desmasificación y la fragmentación, en momentos en que todavía el campo se debate por dejar atrás concepciones ancladas en nociones como la de cultura masiva. Allí se considerarán algunas propuestas que los analistas del campo realizan con vistas a acortar la brecha epistemológica y actualizar las prácticas académicas del campo comunicacional. 


\subsubsection{Objetivos. Dar cuenta de una situación insatisfactoria}

Se procura dar cuenta de la situación actual del ciberperiodismo en las prácticas académicas, considerando el retraso ostensible en la presencia orgánica del ciberperiodismo como objeto de estudio en la investigación y en los planes y programas de estudio, que en la mayoría de los casos se reduce a materias aisladas, no siempre troncales u obligatorias, y a menudo con escasa presencia teórica.

También procura reflexionar sobre las dificultades que podrían interponerse para que la incorporación de los criterios de la nueva cultura de las redes reticulares permee las carreras de periodismo en el grueso de las universidades tomadas en cuenta por la bibliografía y las observaciones aquí consideradas.

Si bien la tendencia es a presentarlo en cursos aislados que se diluyen en medio de concepciones analógicas, han comenzado a surgir iniciativas, como las de la I Jornada Iberoamericana de enseñanza del ciberperiodismo en las Facultades de Comunicación (2012), que reclaman "espacios de reflexión permanente" y un nuevo tratamiento curricular.

Este trabajo también se interroga acerca de las razones que podrían operar para que cuestiones cruciales como la comprensión y metabolización de nociones, como las de las mediaciones y las hipermediaciones, no terminen de incorporarse al acervo de las escuelas de comunicación en toda su fertilidad; cuál es el eslabón de la cadena de acumulación que interrumpe la articulación entre los avances de la teoría y los planes de estudio de las carreras de comunicación en general y de periodismo en particular.

Finalmente, se procurará establecer algunas de las consecuencias sociales que depara esta inconsecuencia académica.

\subsubsection{Metodología. Observando planes de estudio}

Este trabajo se basa en el relevamiento de la bibliografía especializada, en las conclusiones a que arribaron dos investigadores (Said, 2010; Mellado, 2007 y 2010) y en una observación descriptiva (no exhaustiva y necesariamente acotada a la duración de un cuatrimestre) sobre los planes de estudio de la carrera de Periodismo en cuatro universidades uruguayas.

La observación propia se llevó a cabo en mi curso de Nuevas Tecnologías, del quinto semestre de la licenciatura en Comunicación periodística de la Universidad ORT Uruguay, sobre los programas académicos de las cuatro universidades uruguayas que ofrecen cursos de Periodismo, y revela que, como se preveía, que las materias relativas a ciberperiodismo son materias aisladas, desconectadas entre sí, y a menudo electivas y no troncales, que finalmente se diluyen en carreras de concepción analógica.

Buena parte de las preguntas e hipótesis que motivaron este trabajo se generaron en los doce años ininterrumpidos que llevo dictando un curso de Periodismo Digital, que se encuadra perfectamente dentro de muchas de las afirmaciones que aquí se realizan. 
Analizar retrospectivamente la historia del programa (que cambiaba año a año en busca del equilibrio, imposible de lograr para un curso, entre los diferentes aspectos de un escenario que sólo se podría abarcar a través de nuevo plan de estudios) constituyó el primer paso para analizar una inadecuación ostensible.

\subsubsection{Hipótesis de trabajo}

La hipótesis principal de este trabajo consiste en sostener que las dificultades para incorporar la acumulación teórica a los planes de estudio y programas académicos de periodismo en el campo comunicacional, se relacionan más con dificultades del propio campo en propiciar debates abiertos y frontales para superar nociones perimidas, cuya vigencia sólo puede detener el avance del campo comunicacional, que con impedimentos reales vinculados a la especificidad del ciberperiodismo, por ejemplo, las dimensiones ontológicas del cambio operado.

Mientras la academia no termina de formar adecuadamente a sus profesionales, el conjunto social presenta un grado de avance sociotécnico que supera lo que el sistema académico le proporciona. O sea que los usuarios modifican los usos de los dispositivos y son modificados a su vez por ellos, sin que se comprenda del todo qué es lo que se hace y por qué es posible hacer eso que se hace.

La ineficacia de la mayoría de los cursos de periodismo digital radica en que suelen formar parte de carreras pensadas con concepciones analógicas, que sólo se modifican parcial e insuficientemente en adaptaciones que ocurren en ciclos variables sin abordar los cambios en términos de ecosistema.

\section{Un campo disciplinar en crisis}

Según Brownel, un campo disciplinar se constituye en torno a una "comunidad de personas, expresión de imaginación humana, un dominio, una tradición, una estructura sintáctica, un modo de preguntar, una estructura conceptual, un lenguaje especializado u otro sistema de símbolos, una herencia de literatura y artefactos, y una herramienta de comunicación, una instancia evaluativa y afectiva, y una comunidad instructiva" (ápud Coiçaud, 2008: 27).

Pero si bien el modelo de producción de conocimiento, basado en disciplinas, es el que casi todas las universidades importaron, también es, según Gibbons (1998: 5-6), el que comienza a ser superado por formas más eficientes de producción de conocimiento. El tipo de estructura disciplinar

"da la pauta a los investigadores de cuáles son los problemas importantes, cómo se los debe abordar, quién ha de hacerlo y qué se considerará una contribución en esta esfera [...] Llamaremos a esto Modalidad 1 de producción de conocimiento [pero] hay ya pruebas suficientes que revelan que está comenzando a surgir un conjunto nuevo y distinto de prácticas cognitivas y sociales [...] En la Modalidad 1 los problemas se plantean y solucionan 
en el contexto regido por los intereses -principalmente académicos- de una comunidad específica. En cambio, en la Modalidad 2, el conocimiento se produce en un contexto de aplicación [...] es transdisciplinaria [...] se caracteriza por su heterogeneidad [...] posee una jerarquía más plana que usa estructuras organizacionales transitorias [...] tiene mayor responsabilidad social y reflexiva [e] implica un sistema mucho más amplio de control de calidad [...] Indudablemente sigue habiendo evaluación colegiada, pero la Modalidad 2 incluye un conjunto de ejecutantes más amplio, temporario y heterogéneo, que colaboran en un problema definido en un contexto específico y localizado".

Es probable que las deficiencias que los críticos señalan hacia quienes estudiaron el campo comunicacional en las últimas décadas, sin detectar sus debilidades conceptuales, se inscriban en este proceso radical de cambio universitario hacia formatos de producción de conocimiento distribuido, en que la innovación más trascendente ha sido la interdependencia mutua entre el campo de la acumulación teórica y el de la reproducción y divulgación del conocimiento.

Precisamente esa disrupción entre teoría y práctica, que aqueja al campo comunicacional y que la teoría contemporánea de producción de conocimiento virtualmente desaconseja, es la que paradojalmente no fue registrada por los investigadores que se abocaron a relevarlo en las últimas décadas.

Vasallo y Fuentes (2000) se referían, hace más de una década, "a la escasa reflexión que ha existido acerca de la institucionalización de los estudios de comunicación en América Latina y, en ese marco, al escaso interés concedido a los mecanismos y procesos institucionales que intervienen en el desarrollo de las investigaciones que podríamos llamar 'comunicativas' o 'comunicacionales"'. Si bien se han escrito obras de sistematización del pensamiento comunicacional en la región, como las de Jorge Rivera (Argentina), José Marques de Melo (Brasil), Luis Peirano (Perú), Giselle Munizaga (Chile), Ramiro Beltrán (Bolivia), estas "no abordaron suficientemente las razones sustantivas -incluyendo los fundamentos teóricos y las constricciones académico-administrativas- por las cuales, como indican Vasallo y Fuentes, se producen los procesos de elección de los 'objetos de investigación"” (Mata, 2005: 14).

Las observaciones críticas van incluso más allá. Indican que el campo comunicacional regional no sólo desvincula teoría y práctica, sino que tampoco favorece la convergencia en foros comunes de académicos de los diferentes países. Un estudio de 2010, con 35 entrevistas a académicos de la región, lleva a Mellado (2010: 284) a señalar el "notorio desconocimiento por parte de los investigadores en cuanto a la realidad del periodismo y desarrollo de la comunicación social en otros países latinoamericanos":

"Llama fuertemente la atención que más de la mitad de los entrevistados expresen no estar al tanto de las perspectivas conceptuales y modelos de formación del área, más allá de las fronteras nacionales". 


\subsection{Un giro epistemológico no metabolizado}

Una primera consecuencia visible de aquel funcionamiento disruptivo puede detectarse en los alcances de la acumulación teórica comunicacional que permanecen sin incorporarse al acervo y a las prácticas académicas.

En la década de 1980, Verón y Martín-Barbero introdujeron nociones que instaban a trasladar el eje del análisis hacia las mediaciones y superar las concepciones fijadas en los medios, a los que por otra parte, se reducía a meros vehículos transportadores de contenidos. Eran los movimientos teóricos previos a los grandes cambios introducidos en la comunicación en la década de 1990 por la irrupción de las (entonces sí) nuevas tecnologías, con Internet en 1990 y el ciberperiodismo, generalizado a partir de 1994. Ya en ese momento habría empezado a manifestarse una brecha epistemológica que no cesa de profundizarse.

La incomprensión de ese giro de los años 80, dificultó la problematización de "las prácticas comunicacionales abiertas por los nuevos dispositivos tecnológicos [...] tanto las construcciones teóricas que lo constituyen y que se expresan en prácticas de investigación y enseñanza, como esas mismas prácticas académicas" (Mata, 2005: 14). Lo incomprendido, para la mencionada autora, fue el corte producido en los 80 , en que "se fisuró, desde diversos lugares conceptuales, disciplinarios y políticos, esa suerte de 'unidad' [...] entre los medios masivos y la cultura masiva" (ibídem: 15).

En los 90, la noción de sociedad mediatizada proveyó “un nuevo principio de comprensión de los procesos de producción colectiva de sentido en las sociedades postindustriales, una capacidad comprensiva que anteriores categorías -como las de cultura masiva o industria cultural- parecían haber perdido" (ibídem: 16).

Quizá radiquen aquí algunas de las causas que anclan la situación del campo a nociones vencidas que impiden pensar lo nuevo con las categorías que reclama lo nuevo. En el prólogo al clásico de Martín-Barbero ya citado, García Canclini (1991) le apuntaba a las concepciones ingenuas que siguen pensando a los medios en términos de manipulación de audiencias (ese aristocratismo heredado de Frankfurt y cierto marxismo decimonónico). Dicho autor señalaba que en realidad, desde una visión menos ingenua, se advierte que las sociedades cambian cuando irrumpen tecnologías novedosas y lo hacen a través de "la escuela y la iglesia, la literatura de cordel y el melodrama, la organización masiva de la producción industrial y del espacio urbano" (ibidem: 5).

El núcleo resistente con el que debatían Martín-Barbero y García Canclini parece haber sobrevivido y llega hasta nosotros, 20 años después, con muy buena salud y grandes posibilidades de obstaculizar la renovación y la innovación.

\subsection{Reducir la profesión al reporteo y no incidir en la gestión de la información}

Mellado y otros (2007: 156) señalaban, desde otro ángulo, la crisis del subcampo disciplinar del periodismo en la forma de encarar las prácticas académicas: 
“La definición de la profesión, restringiéndola solamente al ámbito del reporteo, es a nuestro juicio caer en un error y en una ingenuidad intelectual, que sólo retrasa e impide un mayor desarrollo de la disciplina a través de la gestión de la información, dando espacio para que actores ajenos a nuestro expertise se integren dentro del ámbito propio de la comunicación social”.

Es que si se reduce el papel de los medios a meros transportadores de textos, operación que prescinde de las mediaciones, se elude precisamente el núcleo de la situación comunicacional contemporánea, constituidas por los procedimientos mediadores que se realizan desde los medios. Como ha señalado Verón (2005: 194), el término "medio" señala un concepto sociológico y no tecnológico:

"No solamente un dispositivo tecnológico particular (por ejemplo, la producción de imágenes y de sonidos en un soporte magnético, sino la conjunción de un soporte y de un sistema de prácticas de utilización (producción/reconocimiento). El video doméstico, que conduce al registro de escenas de la vida familiar, y la televisión de audiencia masiva no se diferencian debido a la naturaleza del dispositivo tecnológico; pero no se trata realmente, en un caso u otro, del mismo 'medio'. Los diferentes procedimientos que confluyen a lo escrito impreso, no son sino dispositivos técnicos. La prensa escrita de lectura masiva es un medio; el equipo computador personal-impresor que ha hecho entrar a lo escrito impreso en el universo de los usos individualizados está probablemente creando otro medio, absolutamente nuevo. El 'medio de comunicación' es por lo tanto para mí un concepto sociológico y no tecnológico”.

Es decir que las prácticas de quienes editan, producen y administran los medios, deben observarse en el escenario crecientemente interactivo en el que juegan unas audiencias dinámicas que establecen un relacionamiento en el que generalmente juegan tensiones y conflictos entre quienes desean que algunas historias se conozcan, y otros igualmente interesados en que esas mismas u otras historias se ignoren. Esa ida y vuelta tradicional, histórico, de la información, está sometido a condiciones cada vez más aleatorias y menos controlables por cualquier poder que respete las mínimas garantías de la libertad de expresión.

Seguir poniendo en juego las nociones como cultura de masas e industria cultural y correlativamente reducir a los medios a transportadores de contenidos en presuntas superestructuras, parece funcional a quienes esquematizan una realidad escasamente verosímil en la que unos medios todopoderosos modelan a la sociedad y pretenden que se acepte colectivamente la visión de que la comunicación consiste en un campo de batalla de unos medios contra otros medios, como si se tratara de partidos. En realidad, "la mediación de los medios se realiza más a gusto del público cuando menos puede hablarse de coincidencia entre quienes administran el poder político y quienes administran la influencia social" (Gomis, 1991: 177).

Sin considerar el concepto de mediación para administrar, el cual la sociedad legitima a medios y periodistas, las operaciones que contribuyen a la construcción social de sentido 
se tornan inapresables e incomprensibles para los profesionales del periodismo y las audiencias. Martín-Barbero (1997: 203) lo decía de este modo:

"Cargada tanto por los procesos de trasnacionalización como por la emergencia de sujetos sociales e identidades culturales nuevas, la comunicación se está convirtiendo en un espacio estratégico desde el que pensar los bloqueos y las contradicciones que dinamizan estas sociedades-encrucijada, a medio camino entre un subdesarrollo acelerado y una modernización compulsiva. De ahí que el eje del debate se desplace de los medios a las mediaciones, esto es, a las articulaciones entre prácticas de comunicación y movimientos sociales, a las diferentes temporalidades y la pluralidad de matrices culturales".

\subsection{El poder de las viejas concepciones "superadas"}

Al cancelar paulatinamente sus capacidades para abordar situaciones comunicacionales de mayor complejidad, el campo disciplinar queda a expensas de reduccionismos y simplificaciones. De ahí que las Facultades y Escuelas de Periodismo "hayan terminado por aportar poco en términos del desarrollo propio del área, convirtiéndose en meras espectadoras de una realidad y de una diversificación laboral que, al parecer, no piensa esperarlas, pero que sí necesita urgentemente de su intervención" (Mellado y otros, 2007: 159-60). Si ya se arrastraban inconsecuencias por incomprensión de nociones como la de mediación, la irrupción de las nuevas tecnologías provoca cambios en cadena que se tornan exponenciales con la introducción de la hipertextualidad y su correlato mediático de las hipermediaciones. Retomaré este tema más adelante.

Si como postula Castells (2012: 23), "la lucha de poder fundamental es la batalla por la construcción de significados en las mentes", no debería eludirse la atribución de intencionalidad a la pretensión de ignorar el conocimiento acumulado en el campo comunicacional. De hecho, el bloqueo de las nociones de las décadas de los 80 y 90, permite a las viejas concepciones que creíamos superadas, cobrar vida en debates cruciales y muy actuales, por esa "construcción de significado".

Como ha señalado Verón (1998: 25), siempre estamos inmersos en "lo ideológico", que no es necesariamente "algo malo: ilusión, error, deformación de clase, prehistoria u obstáculo", sino una "dimensión estructural de toda práctica". De modo que si lo ideológico no es un componente abstracto, aparte, sino que toda noción lo contiene como una dimensión inherente, la prolongación del estatus que desarticula la teoría de las prácticas académicas, de hecho conduce a estas a organizarse en base a concepciones perimidas que sólo pueden irradiar obsolescencia.

Quizá haya que preguntarse a qué segmentos de la realidad regional podría beneficiar, que la academia forme unos profesionales de la comunicación con limitaciones cognitivas relativas a los dispositivos de última generación y sus capacidades operativas, y contrario sensu, a qué segmentos podría perjudicar que las prácticas académicas asuman la acumulación teórica vigente. "La mayoría de los programas académicos profesionales en Comunicación [...] han sido cuidadosos en no reducir su tarea a la enseñanza de oficios y prácticas, en no caer en la 
trampa exclusiva de la reproducción del mundo laboral en las aulas" (Pereira, 2005: 9), pero quizá por cuidarse en exceso de los peligros del mercado, esos mismos planes y programas hayan debilitado sus defensas frente a otras trampas posibles.

Martín-Barbero (1987: 9-10) recordaba que en 1977 "algunos comenzamos a sospechar de aquella imagen del proceso en la que no cabían más figuras que las estratagemas del dominador" y los receptores dominados:

“Justo por esos años algo se nos movió en realidad -por estas latitudes los terremotos no son infrecuentes- tan fuertemente que dejó al aire y nos hizo visible el profundo desencuentro entre método y situación: todo lo que del modo en que las gentes producen el sentido de su vida, del modo en que se comunican y usan los medios, no cabía en el esquema".

Mellado y otros (2007: 157) también abundan sobre el punto que mantiene al campo comunicacional trabado en sus contradicciones y sostienen que en el caso chileno (pero la afirmación, dicen, podría extenderse a la región) "la industria y las organizaciones vinculadas a la gestión de la información han avanzado movidas casi exclusivamente por la innovación tecnológica y los cambios sociales provocados por la llegada del nuevo siglo, y no por la acción de la academia”. Basados en Moragas (2005), los autores consideran que:

"Frente al aumento de la oferta y demanda universitaria en el área del periodismo y la comunicación social, se han cometido grandes errores, que aunque el autor los remite a España, nosotros los enfocamos contextualizadamente a Chile y, presumiblemente a gran parte de Latinoamérica. Dichos errores consistirían, en primer lugar, en la creación de centros universitarios de comunicación sin ningún programa de formación orientado desde el propio proceso de reflexión de la disciplina, sino considerando erróneamente que un cambio de demanda externa justifica la substitución de la formación humanística y social de base, por una prematura formación práctica en comunicación sin mayor análisis previo. En segundo lugar, el inmovilismo de los planes de estudio y su prácticamente igualdad en cualquiera de las universidades chilenas, así como la tendencia a aislar los estudios de comunicación de las propias ciencias sociales y las humanidades, se ha ido traduciendo en puntos débiles de la formación y también de la investigación en comunicación, dando pie a que las propias disciplinas afines a nuestro quehacer, pongan en duda la viabilidad de nuestra profesión y la trascendencia de los estudios en comunicación" (ápud Mellado y otros, 2007: 159).

La desconexión entre la acumulación teórica y el plano de la reproducción del conocimiento académico tendría, como una de las fuentes posibles, el error de no insertar la producción académica en las series de las ciencias sociales y sustituirlas por la "prematura formación práctica en comunicación". Esta hipótesis parece insertar los problemas del campo en la dirección señalada por Gibbons (1998), quien postula que la modalidad 2 de producción de conocimiento académico reclama para sí una mayor flexibilidad que permita acumular en términos transdisciplinarios. 


\subsection{Chile, Colombia, Uruguay. El desconocimiento académico mutuo}

Si las consecuencias de esta desestructuración del campo comunicacional pueden rastrearse hasta el giro epistemológico de los 80 y en la relativa pasividad de las escuelas de comunicación (para incorporar los cambios culturales suscitados por las novedades tecnológicas), así como en cierta reducción de la profesión periodística al reporteo (que prescinde de incidir en la gestión informativa), las deficiencias no pueden dejar de manifestarse en las observaciones realizadas en algunos de nuestros países.

Dos investigaciones de Mellado (2007 y 2010), la primera de ellas citada reiteradamente, y otra de Said (2010) coinciden con mi propia observación, menos ambiciosa y acotada a la presencia del periodismo digital en los planes de estudio de Periodismo en las universidades uruguayas (2013), en la débil presencia del ciberperiodismo en el campo comunicacional de la región.

En el caso chileno, Mellado señala que "la industria y las organizaciones vinculadas a la gestión de la información han avanzado movidas casi exclusivamente por la innovación tecnológica y los cambios sociales provocados por la llegada del nuevo siglo y no por la acción de la academia" (ápud Mellado y otros, 2007: 157). Más adelante la autora (2010: 274) advierte también que la academia latinoamericana "sigue pensando el ejercicio y significado profesional del periodismo a partir de los medios de comunicación". Asimismo, afirma que "las escuelas de periodismo y las facultades de comunicación hoy se enfrentan a la dificultad de unir de manera armoniosa el contenido de las técnicas periodísticas con las bases teóricas y el estudio de la comunicación social" (ibídem: 277).

Para el caso colombiano, Said (2010), coincide en que si bien "los docentes de la Fcs-Col parecen estar conscientes de la importancia dinamizadora que están ejerciendo las TIC en la formación de nuevas tipologías profesionales al interior del periodismo [...] no existen señales de reconocimiento de la complejidad conceptual que encierra el ciberperiodismo".

"Si bien es cierto que el concepto de ciberperiodismo alude también a todo lo que encierra la labor periodística tradicional, la forma como lo definen los docentes no da señales de incluirse otros rasgos que caracterizan el creciente escenario mediático digital [como] el auge en la colaboración entre agentes de información y comunicación; la ampliación de canales de interacción entre periodistas y usuarios [lectores]; el aumento de la participación de estos últimos; así como la adquisición de e-competencias [...] en la nueva generación de profesionales que se están formando [...] los datos aquí mostrados nos muestran a unos docentes que no son capaces de reconocer: 1) la incidencia que tendrá la dinamización de un nuevo modelo comercial y profesional, ante el auge de los medios digitales desde internet; y 2) la incidencia que tiene su labor pedagógica en la formación de estos profesionales, en aspectos vinculados con el fomento del hábito de uso de las TIC y de una cultura digital en estos" (Said, 2010: 41). 
La observación ${ }^{1}$ que se planteó a un equipo de estudiantes de mi curso de Nuevas Tecnologías $\left(5^{\circ}\right.$ semestre de la Licenciatura en Comunicación Periodística, Universidad ORT Uruguay), no se propuso más que determinar la forma en que el ciberperiodismo se insertaba en las carreras de Periodismo de las universidades uruguayas. Sin embargo, los resultados obtenidos, más las entrevistas realizadas a algunos responsables de las carreras respectivas, permite asegurar que el panorama no es muy diferente al enunciado por el análisis de Said para la realidad académica colombiana, citado anteriormente.

En Uruguay hay 4 universidades con carreras de Periodismo y seis materias están vinculadas al ciberperiodismo, de las cuales sólo tres son obligatorias y las tres restantes optativas. Es decir, que entre las 132 materias que en total dictan las cuatro universidades que ofrecen carreras de periodismo en Uruguay, seis están vinculadas al ciberperiodismo, y únicamente tres, es decir el 0,02 por ciento, son obligatorias.

El extremo lo expresa la Licenciatura en Comunicación de la Universidad de la República (que se desarrolla actualmente con un plan de 1995) cuenta con 26 materias y no tiene ninguna materia vinculada a ciberperiodismo. La Universidad Católica del Uruguay cuenta con una carrera de Periodismo con 45 materias, de las cuales una está vinculada al ciberperiodismo ( 80 horas, sobre 2200 del total). La materia se denomina Periodismo en Internet, se ubica en el tercer año, es obligatoria y presencial, y sus contenidos son 50 y 50\% teórico-prácticos.

La Universidad de Montevideo tiene una carrera de Periodismo con 15 materias y dos vinculadas al ciberperiodismo: Narrativa online, que se dicta en el sexto semestre, es obligatoria y sus contenidos se distribuyen por partes iguales entre teoría y práctica. A su vez, Diseño, en el quinto semestre, tiene una concepción de producción online y está dedicada en un $20 \%$ a proporcionar teoría y $80 \%$ formación práctica. Es una materia optativa.

La Universidad ORT Uruguay, tiene una carrera de Comunicación Periodística con 46 materias que data de un plan de 2009, entre las cuales hay 3 vinculadas al ciberperiodismo, con 144 horas sobre un total de 3.528 horas de la carrera: Nuevas tecnologías, va en el quinto semestre, es obligatoria y proporciona un $60 \%$ de contenidos teóricos y $40 \%$ de formación práctica. Gestión de la comunicación online se ubica en el sexto semestre, es electiva y proporciona $30 \%$ de contenidos teóricos y $70 \%$ de formación práctica. Redacción de Blogs va en el $3^{\circ}$ semestre, es electiva y $100 \%$ práctica.

Como puede advertirse, la presencia del ciberperiodismo en las carreras de periodismo de las universidades uruguayas es apenas incipiente y no llega a dar cuenta (por la propia desconexión de los cursos dentro de cada carrera y por la predominancia de las competencias prácticas sobre la teoría) de las complejidades que reclama la acumulación teórica.

1 En esta observación (que se desarrolló entre marzo y junio de 2013) participó un equipo de trabajo integrado por los estudiantes del $5^{\circ}$ semestre de Periodismo: Sofia Drago, Martín Fernández, Santiago Pereira, Bernardo Wolloch y el Lic. Tomer Urwicz. 
Para decirlo con palabras de Said (2010: 41), ni en Colombia, ni en Chile ni en Uruguay, parecen existir "señales de reconocimiento de la complejidad conceptual que encierra el ciberperiodismo".

\section{El desacople teórico-práctico contribuye a construir una realidad distorsionada}

Verón escribía en 1987 que el término "mediatización" había "saltado de los ámbitos académicos" hacia los medios que comenzaron a "utilizarlo frecuentemente para hablar de sí mismos". Desde la perspectiva actual debe precisarse que el concepto saltó a los medios pero no desde los medios académicos, sino desde la teoría, ya que si bien se lo ha utilizado en algunos programas académicos, la noción todavía no está incorporada fehacientemente al acervo de las prácticas del campo, por las razones en las que se viene insistiendo a lo largo de este artículo.

Según el autor, también podía deducirse que "el término se ha incorporado ya a la vieja familia de los 'operadores semánticos', destinados, en el discurso de los medios a generar un sentimiento de comprensión de las situaciones a las que se aplican ( $c f$. Verón, 1969) 'imperialismo', 'colonialismo', ayer; 'mediatización', 'globalización', hoy; 'crisis', siempre” (ibídem, 1987).

Sin embargo, parecería que la operación que condujo el término a los medios no se realizó en forma consciente. Con frecuencia, la comprensión de los procesos globales resulta alterada por la inadecuada percepción que tiende a homogeneizar sus manifestaciones, igualándolos en sus efectos en todas las realidades, como si sus consecuencias fueran similares y pudieran homologarse con prescindencia de factores conexos y que a menudo relativizan o atenúan, cuando no distorsionan, los efectos de los fenómenos innovadores.

No sería la primera vez que adoptamos respuestas para problemas cuyas preguntas no han sido formuladas, una típica cuestión de corte epistemológico que constituye una rémora del atraso y que a su vez opera en forma reproductora de esas condiciones. Quizá la crisis del campo comunicacional a que se refieren los análisis aquí considerados, también se manifieste en los países desarrollados, pero la realidad de los países iberoamericanos no debería asimilarse ligeramente con las de sociedades que, por sus propios méritos, crean y desarrollan las tecnologías que revolucionan las formas de producir bienes y servicios. Quien innova, cuenta (así ha sido históricamente) con algo más que tiempo a su favor; cuenta nada menos que con la capacidad de comprensión social de aquello que surgió de su seno.

Las redes reticulares y el ciberperiodismo surgieron y se propagaran en sociedades en que el conjunto de los operadores sociales (la academia entre ellos) se fue organizando simultáneamente en forma digital. Ese acompasamiento generalizado contribuye a la comprensión colectiva de un modo que puede suplir o amortiguar (si es que existieran) eventuales retrasos en la divulgación académica. El atraso de nuestros países, que por lo general acceden a las tecnologías de punta con retrasos y ritmos propios de la escasez de recursos (por algo somos históricamente tomadores de conocimiento, tecnologías, precios) se manifiesta, entre otras cosas, por las desigualdades y asimetrías de su propio desarrollo interno. 
En definitiva, probablemente se hable y se mencione exageradamente el término mediatización, pero ello no parece tener demasiada relación, no al menos una directa, con que se lo comprenda cabalmente. Quizá sea de utilidad recurrir a algunos ejemplos.

\subsection{La academia, los públicos, las audiencias interactivas. Realidades desiguales}

Como en general lo obvio resulta complejo de registrar, si la academia no incorpora en sus prácticas las nociones que la teoría describió en la década de 1980 y 1990, parece lógico suponer que el público, e incluso sus segmentos dinámicos, puedan quedar bajo la hegemonía de nociones que datan de los comienzos de la teoría de la comunicación, en las primeras décadas del siglo XX. Sin embargo, esos mismos públicos, en tanto usuarios, y pese al lastre de "pensar" con nociones obsoletas, acometen con espontánea elasticidad el uso de los nuevos dispositivos y asumen con naturalidad sus consecuencias sociotécnicas. Es decir, que modifican simultáneamente sus usos y sus propios comportamientos a partir de esos usos.

Algunos segmentos de usuarios (se piensa en los jóvenes pero no son sólo ellos, aun cuando sean principalmente ellos) se encuentran en el presente, en lo relativo a usos de los dispositivos tecnológicos. Esas audiencias dinámicas e interactivas (generadoras de circuitos y comunidades virtuales, que consumen los medios e interactúan con estos desde las redes sociales) se encuentran desde el punto de vista de sus prácticas, en 2013.

Los viejos medios tradicionales, ya inevitablemente contaminados por la cibercultura y los nuevos medios surgidos en la lógica de las redes reticulares, son utilizados por estas con naturalidad y eficiencia. Dichas prácticas, con sus modificaciones actitudinales, incluyen a los propios profesionales de la comunicación, quienes aun sin recibir formación específica, deben adaptarse intuitivamente a las nuevas condiciones y responder a los requerimientos de los públicos ya transformados en generadores de información. Pero esos mismos usuarios no tienen por qué advertir la incongruencia de utilizar dispositivos digitales e interactuar en redes sociales que operan la reticularidad descentralizada, al tiempo que razonan en términos de medios con capacidad de manipulación de audiencias indefensas.

Creo haber puesto de manifiesto estos desniveles cognitivos en mi artículo "La mediatización insuficiente es funcional al atraso" (2009), donde procuré relevar ejemplos en los cuales la producción periodística incurre en inconsecuencias propias de la falta de comprensión de nociones que el campo comunicacional ha definido, pero a las cuales la academia no ha otorgado la difusión adecuada. En consecuencia, la sociedad no se las apropia, estableciéndose un déficit en el plano de la mediatización que no puede manifestarse sino como desarreglos de la circulación normal de la información.

\subsection{La mediatización insuficiente es funcional al atraso}

Los medios del viejo ecosistema atraviesan (desde hace por lo menos dos décadas) un escenario problemático. Uno de los equívocos radica en reducir la crisis a un modelo de negocios como si no fuera constitutiva del modelo informativo. Pero lo es y afecta al formato (monologal, 
estructuralmente vertical, autoritario y cerrado) en que se ha producido, distribuido y consumido la información desde el surgimiento de la sociedad industrial.

En 2009, a partir de una pregunta de la revista argentina LIS (Letra, Imagen, Sonido) sobre la situación y perspectivas de los medios uruguayos en el horizonte de la década siguiente (2019), escribí que los medios (no uno sino el conjunto de ellos) incurren con relativa habitualidad en la subestimación u omisión de historias que entrañan cierta relevancia, y al mismo tiempo sobreestiman y sobreexponen asuntos de enorme banalidad.

No es que exista un plan perverso de ocultamiento y desinformación (digámoslo para quienes pretendan ver ratificadas sus presunciones de atribución de propósitos de manipulación mediática) sino que, por diversas razones, en ocasiones, un tema relevante no se serializa adecuadamente. Un modelo posible de este tipo de problema podría ser el escándalo Lance Armstrong. Toda la afición al ciclismo sabía o al menos sospechaba la presencia del doping, pero el periodismo deportivo "tabuizaba" el tema; era de lo que no se hablaba. Cuando estalló el escándalo, sólo podía escandalizar a los propios medios. Cuando el público sabe más que lo que los medios le informan se dan situaciones mediáticamente insostenibles por las cuales el ecosistema paga costos altos que a menudo los propios medios no perciben.

La pregunta sería: ¿Por qué desciende lo relevante y se incrementa la banalidad? Y la respuesta resulta bastante obvia. En términos de costos, puede parecer más fácil, y por tanto más económico, que los públicos masivos compren un discurso con escasa exigencia, que demanda poca información previa, que a la inversa. Así parecen afrontar la crisis muchos de los medios del viejo ecosistema; con cambios mínimos y vendiendo banalidad. Mi pronóstico para la década siguiente no era el más positivo, pero no es lo que aquí importa.

Simultáneamente, importantes medios de referencia (no ya uruguayos, sino globales) incurrían en graves inconsecuencias y errores que también evidenciaban dificultades del ecosistema, ya que desde el caso Jayson Blair al escándalo de escándalos de News of the World, de Rupert Murdoch, no podían cargarse ligeramente a negligencias o inoperancia de sus editores y/o periodistas.

Incluso debe señalarse que los escándalos mencionados, todos ocurridos en medios occidentales, y por tanto en "sociedades abiertas", resultan incomparablemente menos significativos que el escándalo perpetuo de los sistemas mediáticos de las "sociedades cerradas", con pensamiento hegemónico o directamente totalitario. Es el caso de Cuba o Corea del Norte, donde los escasísimos medios responden a decisiones burocráticas del gobernante de turno, o Rusia, China o Irán, donde los medios se encuentran bajo tutela. En varios países hispanoamericanos, los medios se encuentran en situaciones altamente inestables, bajo fuertes presiones cuando no aceptan someterse a poderes que los interpelan en términos de aquellas nociones perimidas que la academia no ha dado colectivamente por obsoletas.

Pese a sus diferencias, todas estas realidades (ya sean las de sociedades abiertas como las de sociedades cerradas) resultan de un mismo denominador común que unifica a todos los sistemas de medios surgidos en la era industrial. Los ha puesto en jaque el nuevo ecosistema basado en 
redes reticulares. Con ese adversario invisible no pudo Murdoch con sus prácticas de espionaje de su propio lectorado que lo llevó al cierre abrupto de un medio con más de siete millones de compradores, ni tampoco el régimen de los hermanos Castro, que le temió durante una década a una pequeña y frágil bloguera a la que no ha tenido otro remedio que metabolizar.

Todas estas cuestiones, inexplicadas, y por tanto no comprendidas colectivamente, tienden a conformar el ruido que habitualmente se decodifica con la paradoja de la desinformación en medio de la sobreinformación. Esta situación la ha señalado con claridad Bolz (2006: 92-93):

"Nuestros grandes problemas no resultan de una falta de conocimiento, sino de orientación. No somos ignorantes, estamos confundidos, pero esto se tapa con el entusiasmo de la 'era de la información' y sus hechos. Bajo la presión de las nuevas tecnologías, nos inclinamos a interpretar todos los problemas como problemas de desconocimiento. Sin embargo, las cuestiones de sentido y los problemas de orientación no se resuelven con informaciones".

No obstante, en términos periodísticos, la teoría consolidada indica que la repetición de los temas (lo que se conoce como "redundancia mediática" en el ecosistema industrial) "un día y otro día es el más potente de todos los mensajes para que nos quede clara su importancia". Énfasis u omisiones del conjunto de los medios, hacen que la agenda periodística "se vuelva en gran medida, la agenda pública" (McCombs, 2006: 25). En buen romance, aquellos sobre lo cual los medios (el conjunto de ellos, diarios, radio, televisión) insisten, se transforma en agenda pública. Aquello sobre lo cual no se abunda, se pierde en el olvido.

"No es que la sociedad sea cada vez más compleja", dice Judith Schlanger, "es el saber que tenemos sobre el mundo el que al aumentar permite descubrir, de manera ciertamente paradójica, su complejidad. Esto es y será verdadero en todas las épocas" (ápud Charaudeau, 2003: 40). Pero cuando lo que prevalece no es la mirada compleja, y falta la interrogación pertinente, los actores sociales (políticos, empresariales, sindicales y culturales) andan a su aire, sin rendir cuentas. Puede pasar de todo sin que se releve, ni se lo articule. A una sociedad así le pasan cosas, pero no aprende; tropieza con los obstáculos, pero no elabora. En consecuencia, sus medios se empobrecen (no sólo económicamente) y juegan un papel cada día más débil (Mazzone, 2009).

Ese descenso discursivo diluye no sólo la mediatización, sino también la calidad de la vida democrática y de las instituciones, y cabe la pregunta acerca de si ocurriría lo mismo con una academia que no permaneciera como espectadora no participante. Digamos de paso que analizar los problemas del periodismo, en términos de redundancia dentro del ecosistema industrial, es también una metodología que va quedando desactualizada, en la medida en que el nuevo ecosistema tiende (como veremos) a la desmasificación y la fragmentación. En las nuevas condiciones no resultará de mucha utilidad seguir pensando en términos de mediaciones y audiencias masivas.

Señala Castells (2012: 23): "Para la sociedad en sentido amplio, la principal fuente de producción social de significado es el proceso de comunicación socializada (o sea) aquella que existe en el ámbito público más allá de la comunicación interpersonal". Es por tanto un tema que se abre 
para la academia, el de la investigación y análisis de la agenda mediática y pública, en el marco de las nuevas audiencias en movimiento, con capacidad de generación de información.

\subsection{El tipo de problema que la academia debería explicar}

Cuando Scolari y Castells sugieren ir más allá de las pantallas a observar las transformaciones y los fenómenos sociales, producidos a partir de las hipermediaciones y la autocomunicación de masas, se advierte que nos encontramos en sociedades (también las iberoamericanas, o al menos sus segmentos más activos) que están en condiciones de comprender los nuevos fenómenos de la comunicación, dado que se encuentran inmersos en ellos, los protagonizan.

La academia no debería eludir la explicación (no porque no sea complejo, sino porque lo es) acerca de qué ocurre cuando el flujo normal de la comunicación se interrumpe o distorsiona, por acción u omisión de uno o varios agentes del ecosistema de medios. Explicar, divulgar, equivale a incluir razonablemente en los planes y programas de enseñanza (desde el enfoque de las carreras de comunicación) las nociones teóricas fundamentales que conducen a construir sentido en los procesos comunicacionales contemporáneos.

Si a la ciudadanía le resulta sencillo advertir que un corte generalizado del agua o de la energía eléctrica o del transporte en una ciudad resulta altamente perjudicial para el funcionamiento colectivo, es porque puede sentir, en forma directa y tangible, la ausencia del flujo que nutre los circuitos que aseguran la normalidad en cualquiera de esos campos. La pregunta es por qué no se registra del mismo modo (natural y espontáneo) los altibajos, las interrupciones y disrupciones en el suministro de la información.

Se dirá que no se puede comparar la simplicidad de la comprobación (directa, tangible) en los campos mencionados, con la complejidad del flujo informativo (abstracto) en el campo comunicacional. Sin embargo, lo que no es de recibo argumentar es que el acceso a la información de calidad no asume (en la sociedad actual) la importancia crucial del suministro normal de agua, energía o transporte.

Admitida la importancia del flujo informativo para la vida social, el verdadero problema se ubica entonces, en el plano de cómo proveer explicaciones complejas. Pero acaso explicar la comunicación no puede ser más abstruso que explicar las razones de una interrupción en el flujo energético. Quizá este sea uno de los desafíos a tomar por parte de los teóricos de la comunicación y el sistema académico: terminar de instalar, en la práctica concreta de todos los días, la comprensión de los problemas cuya interferencia modifica, a veces imperceptiblemente, la vida de cada uno de nosotros.

\section{Hacia la desmasificación y fragmentación. Dos efectos del nuevo ecosistema}

Mientras las mediaciones jugaban en entornos definidos por las fronteras nacionales, en un ecosistema de medios surgido con la sociedad industrial, el nuevo y descentralizado 
ecosistema mediático de las redes reticulares no tiene otro límite que sus propios nodos en un escenario que tiende a ser global.

En la nueva trama de las hipermediaciones, Scolari (2008) señala que el usuario, hasta por el sólo hecho de navegar y establecer contactos con una gran diversidad diaria de páginas web, mueve las agujas del page rank y por tanto jerarquiza, aun inconscientemente, unos hipertextos frente a otros. El desafío que tiene por delante el campo comunicacional es determinar de qué forma se actualiza para abordar estos nuevos tiempos en que prevalecerán la desmasificación y la fragmentación, cuando todavía existen resistencias deterministas que pretenden remitir las prácticas académicas a las nociones de la masividad y la homogeneidad de textos ordenados según la secuencialidad.

Mata (2005) ha atribuido la permanencia de "visiones instrumentales y deterministas que impiden una cabal comprensión de los procesos de producción de la cultura contemporánea" al "débil trabajo conceptual y metodológico" que ha desestimado las "renovaciones teóricas de trascendencia producidas en las últimas dos décadas del siglo pasado". El problema para el campo comunicacional reside entonces en cómo pasar de la hegemonía de las visiones instrumentales y deterministas al análisis de "la trama de reenvíos, hibridaciones y contaminaciones que la tecnología digital [introduce en] configuraciones que van más allá -por encima- de los medios tradicionales" (Scolari, 2008: 114).

\subsection{De las mediaciones a las hipermediaciones y a las transformaciones sociales}

El problema sigue siendo el desfase entre la acumulación teórica y los programas académicos, que revela una distancia distorsionante entre las definiciones teóricas y las formas de resolver los problemas que demanda la práctica académica. Si este es el punto que detiene y retrasa el desarrollo del campo, pues deben determinarse los mecanismos que fallan e impiden cumplir con "la misión de nuestras instituciones [que] consistiría en formar personas que sepan cómo manejarse en esta supercomplejidad, actuando propositivamente en ambientes que se modifican permanentemente" (Coiçaud, 2008: 30).

Tan real es la alusión que nuestros futuros profesionales deben adaptarse de manera constante "en ambientes que se modifican permanentemente", que ya es insuficiente postular la necesidad de pensar en términos de mediaciones, ante el nuevo giro que nos instala en el ambiente de las hipermediaciones.

¿Qué diferencia a las hipermediaciones de las mediaciones?

"La tecnología digital ha potenciado y evidenciado algo que antes existía solo en teoría: la textualidad entendida como red (Bajtín, 1982, 1986; Kristeva, 1978). Por lo que respecta a los receptores, del consumo activo, rebelde y contrahegemónico de las mediaciones entramos en otra dimensión donde el usuario colabora en la producción textual, la creación de enlaces y la jerarquización de la información" (Scolari, 2008: 114). 
No debe caerse en el error de trazar una línea divisoria entre las mediaciones y las hipermediaciones, sino por el contrario, se debe ubicarlas en la misma serie histórica de lo comunicacional:

"Las hipermediaciones no niegan a las mediaciones, sólo miran los procesos comunicacionales desde una perspectiva diferente y los ponen en discurso desde otra perspectiva. Si el estudio de las mediaciones proponía analizar las articulaciones entre las prácticas de comunicación y los movimientos sociales, las investigaciones de las hipermediaciones deberían salir de la pantalla para analizar las transformaciones sociales que el desarrollo de nuevas formas de comunicación está generando" (ibídem: 117).

Por su parte, Castells (2009: 44) trabaja con la hipótesis de que "las configuraciones relativamente estables construidas en las intersecciones de estas redes pueden constituir los límites que podrían redefinir una nueva 'sociedad', teniendo en cuenta que dichos límites son altamente volátiles por el cambio constante de la geometría de las redes globales que estructuran las prácticas y organizaciones sociales".

La teoría induce a pensar en términos de escenarios dinámicos, de configuraciones volátiles, de cambio ininterrumpido hacia la conformación de un nuevo tipo de sociedad de la que, por el momento, sólo sabemos que será una "sociedad-red". Pero de la que debemos suponer que nos exigirá mayor flexibilidad y capacidad de adaptación que la exhibida, si pretendemos ubicarnos a la altura de las demandas que presumiblemente interpelarán a la academia en el futuro próximo.

\subsection{El comienzo de una adaptación posible. Releer, reconsiderar, ¿relanzar?}

La propuesta de Mata (2005) en la cual coinciden Mellado y otros (2007), consiste en releer "la producción conceptual y empírica contenida en las investigaciones que se realizan en el campo de estudios de la comunicación”, para determinar en qué series se insertan:

"Si en las series que analizan los procesos de individuación sistemática iniciadas a partir de la lectura silenciosa y de apropiación individual del objeto libro [o en las] series construidas a partir de interrogaciones en torno a las limitaciones que una determinada moralidad debía y debe fijar para la difusión de contenidos [...] Sin un ejercicio de esta naturaleza, los avances que se realizan desde diversas zonas de los estudios de comunicación, correrán siempre el riesgo de soslayar [...] la acumulación de evidencia conceptual y empírica y seguiremos resignándonos a reproducir un escenario en el cual la yuxtaposición no acumula, la igualación debilita, la creatividad y el riesgo de pensar son cubiertos por la monocorde declinación de tópicos autolegitimadores" (Mata, 2005: 20).

En las múltiples operaciones intermedias que las universidades realizan para adaptar su oferta a las demandas sociales, se registra un débil "trabajo conceptual y metodológico" que resigna posibilidades de aquilatar la acumulación teórica ante la fuerza inercial que desconoce los hallazgos empíricos y su procesamiento ordenado. 
Si la academia ocupa el plano simbólico del conocimiento y, por tanto, el que la sociedad legitima para construir sentido en los procesos sociales, parece de toda lógica presuponer que, a la inversa, si esa capacidad no se ejerce (y los hechos irrumpen sin que se establezcan vínculos cognitivos eficaces) las incomprensiones se transfieren multiplicadas al conjunto social.

De modo que cabe interrogarse acerca de las razones por las cuales si la teoría no vaciló en señalar que el cambio es ontológico, si las prácticas académicas (y aun las declaraciones y reflexiones de no pocos académicos) parecen dejar abierto un espacio para dudar de las evidencias y de hecho creer en la posibilidad de resolver la coyuntura con la incorporación de materias técnicas aisladas.

\subsection{Teorizar es problematizar, confrontar}

Lo que se ha modificado radicalmente es el escenario que permitía ejercer el periodismo con las formas en que nació durante la revolución industrial del siglo XIX y que hizo de la sociedad industrial la primera sociedad mediática. A lo largo de estos casi dos siglos, los medios y el ecosistema se modificaron en forma constante, con tal intensidad que incluso condujeron a la sociedad post-industrial, a la que se define en vías de mediatización o mediatizada, es decir, que cambia precisamente por la existencia de medios.

Si el ecosistema se diversificó (a los diarios se agregaron la radio, la televisión, el cine y finalmente el gran metamedio de Internet) con cambios que lo complejizaron, ninguno de ellos tuvo la dimensión ontológica del arribo de las redes reticulares, que terminó por decretar el fin de la forma industrial de producir bienes y servicios, y también información. Así comienza el más reciente capítulo de la mediatización.

Estas definiciones todavía no han sido admitidas, de hecho, por el grueso de las carreras de periodismo de la región, que suelen ubicar al ciberperiodismo como una asignatura, no sólo retrasando y dificultando la aproximación a la teoría, sino limitando la percepción colectiva, al prolongar la vigencia de categorías que ya no están en condiciones de expresar ni comprender los nuevos fenómenos.

A partir de los cambios introducidos por la reticularidad de las redes, y sobre todo con las profundas modificaciones que pusieron a las audiencias en movimiento, emergieron las redes sociales que transformaron el relacionamiento entre los medios y sus audiencias, así como el vínculo de los ciudadanos con la política. Comprender estos cambios, entender a qué se deben y cómo operarlos (al servicio de mejores condiciones en la circulación de la comunicación) es uno de los grandes temas de la mediatización y, por tanto, de la academia y de los programas de formación de nuevos profesionales.

\section{Conclusiones. De espectadores a protagonistas}

Hace más de una década que se detectó una desarticulación teórico-práctica en el campo comunicacional, que relega a las Escuelas de Periodismo a ser espectadoras de los grandes 
cambios en curso. Sucesivos análisis no dejaron de subrayar el desajuste sin lograr revertirlo, ni instalar el debate en torno a posibles estrategias para afrontarlo. Simultáneamente, el marco académico registró, sobre el final del siglo XX, "el surgimiento de un sistema distribuido de producción de conocimiento" y cuya modificación más profunda, según Gibbons (1998), consiste en que la producción y divulgación del conocimiento (la investigación y la enseñanza) ya no son actividades autónomas, que se llevan a cabo en instituciones relativamente aisladas.

La década de 1980 parece marcar un punto de inflexión con un giro epistemológico que el campo no ha metabolizado satisfactoriamente y se manifiesta en las dificultades para superar la mirada sobre los medios, para pasar a la comprensión de las mediaciones y las complejidades de la mediatización. Esa transición incompleta abrió una brecha cognitiva que el campo comunicacional expresa por ausencia de las nuevas nociones en los objetos de estudio (escogidos para investigar) y en los planes de estudio y programas académicos, que inevitablemente se amplifica en su proyección social. En los debates indirectos y sesgados (que refieren a los medios y a las mediaciones y que vehiculizan los propios medios) reaparecen, más o menos explícitas, percepciones que aluden a amenazas mediáticas en su presunta capacidad para manipular audiencias masivas.

En el uso acelerado y creciente de los nuevos dispositivos digitales (desde los cuales esas mismas audiencias interactúan con medios que no saben qué hacer con esas capacidades interactivas) destaca la escasa inverosimilitud de ese discurso. Esta incomunicación de la comunicación distorsiona la divulgación académica de nociones básicas que permitirían explicar la complejidad mediática y acompasar la percepción colectiva con la espontaneidad sociotécnica de audiencias, que se incorporan en forma creciente a la generación informativa.

Revertir el desacople entre la producción del conocimiento y su divulgación en el campo comunicacional requerirá que los respectivos subcampos (también el periodismo) dejen de proyectarse aisladamente y se adapten a la construcción teórica transdisciplinaria. Mientras el campo sigue anclado en el intento de metabolizar acumulaciones de hace décadas, los nuevos procesos mediáticos continúan introduciendo desafíos. Sin haber terminado de integrar conceptos como el de las mediaciones, la academia debe adecuarse a la nueva realidad de las hipermediaciones con sus efectos de desmasificación y fragmentación.

No alcanza con centrar la enseñanza universitaria en el funcionamiento de las herramientas multimedia, sino inducir a la reflexión y a la elaboración conceptual acerca de las nuevas condiciones en que la información dejó de ser un monólogo para transformarse en una conversación. Y sobre todo ponerse en situación de superar, definitivamente, las ya obsoletas nociones de cultura masiva y las maliciosas percepciones de los medios en términos de manipulación de audiencias que nada pueden significar en tiempos de desmasificación y fragmentación.

En los noventa, la noción de sociedad mediatizada proveyó "un nuevo principio de comprensión de los procesos de producción colectiva de sentido en las sociedades postindustriales, una capacidad comprensiva que anteriores categorías -como las de cultura masiva o industria cultural- parecían haber perdido" (Mata, 2005: 16). 
Retomando la hipótesis principal, y sin dejar de considerar que la producción de conocimiento ingresó en una nueva fase de acumulación transdisciplinaria que demanda nuevas complejidades metodológicas, puede concluirse que será el debate más abierto y frontal uno de los recursos principales hacia el recorte de la brecha entre la acumulación teórica y su divulgación.

\section{Fuentes consultadas}

Bolz, N. (2006). Comunicación mundial. Buenos Aires: Katz.

Castells, M.

_(2012). Redes de indignación y esperanza. Los movimientos sociales en la era de Internet. Madrid: Alianza.

_(2009). Comunicación y poder. Madrid: Alianza.

Charaudeau, P. (2003). El discurso de la información. Barcelona: Gedisa.

Coiçaud, S. (2008). El docente investigador. La investigación y su enseñanza en las universidades. Buenos Aires: Miño y Dávila Editores.

Ferrés i Prats, J. (2003). "Educación en medios y mecanismos de identificación". XXI, Revista de Educación, núm. 5, pp. 51-59.

García Canclini, N. (1991). "Prólogo". En Martín Barbero, J. De los medios a las mediaciones. Barcelona: Gustavo Gili.

Gibbons, M. (1998). "Pertinencia de la educación superior en el siglo XI. Education. The World Bank". Conferencia Mundial sobre la Educación Superior de la UNESCO. París.

Gomis, L. (1991). Teoría del periodismo. Barcelona: Paidós.

I Jornada Iberoamericana (2012). "Declaración conjunta sobre la enseñanza del ciberperiodismo en las facultades de comunicación" (2012, agosto 14). Medellín: Universidad Pontifica Bolivariana de Medellín, Gabinete de Comunicación y Educación de la Universidad Autónoma de Barcelona. Extraída el 23/VIII/2013 desde http://www.upb.edu. co/pls/portal/docs/PAGE/ gpv2_upb_medellin/pgv2_m030_pregrados/pgv2_m030040010_ comunicacion/pgv2_m030040010080_memorias/declaracion_ciberperiodismo_final.pdf

Martín-Barbero, J. (1997). De los medios a las mediaciones. Comunicación, cultura y hegemonía. Barcelona: Gustavo Gili.

Mata, M. (2005). "Los medios masivos en el estudio de la comunicación/cultura”. Conexao - Comunicaçao e Cultura, UCS, vol. 4, num. 8. pp. 13-21. 
Mazzone, D. (2009). "La mediatización insuficiente es funcional al atraso". Revista LIS (Letra, Imagen, Sonido), año 1, núm. 4. Extraída el 23/VIII/2013 desde http:// semioticafernandez. com.ar/revista-lis/articulos-lis-4/

McCombs, M. (2006). Estableciendo la agenda. El impacto de los medios en la opinión pública y el conocimiento. Barcelona: Paidós.

Mellado, C. (2010). "La voz de la academia: Reflexiones sobre periodismo y comunicación". Signo y Pensamiento, vol. XXIX, núm. 56, pp. 274-287.

Mellado, C. y otros (2007). "Investigación y perfiles profesionales en periodismo y comunicación para una actualización curricular permanente”. ZER, núm. 23, pp. 139-164.

Pereira, J. (2005). "La comunicación: un campo de conocimiento en construcción. Reflexiones sobre la comunicación social en Colombia”. Investigación y Desarrollo, vol. 13, núm. 2, pp. 412-441.

Said, E. (2010). "El docente ante el avance del ciberperiodismo en Colombia”. Enl@ce: Revista Venezolana de Información, Tecnología y Conocimiento, vol. 7, núm. 1, pp. 27-44.

Scolari, C. (2008). Hipermediaciones. Elementos para una teoría de la comunicación digital interactiva. Barcelona: Gedisa.

Sodré, M. (1998). Reinventando la cultura. Barcelona: Gedisa.

Vasallo, M. y Fuentes, R. (comps.) (2000). “Comunicación. Campo y objeto de estudio. Perspectivas reflexivas latinoamericanas”. México D. F.: Iteso, Universidad Autónoma de Aguascalientes, Universidad de Colima, Universidad de Guadalajara.

Verón, E.

_(2011). Papeles en el tiempo. Buenos Aires: Paidós.

(2005). Fragmentos de un tejido. Barcelona: Gedisa.

_(1998). La semiosis social. Fragmento de una teoría de la discursividad. Barcelona:

Gedisa.

_(1987). "Esquema para el análisis de la mediatización”. Cursos y conferencias $N^{\circ} 9$. Universidad de Buenos Aires, Facultad de Filosofía y Letras. 